\title{
A wideband profiled corrugated horn for multichroic applications
}

\author{
Lingzhen Zeng ${ }^{1}$, Cheuk-yu Edward Tong ${ }^{1}$, Edward J. Wollack ${ }^{2}$, and David T. Chuss ${ }^{3}$ \\ ${ }^{1}$ Harvard-Smithsonian Center for Astrophysics, Cambridge, MA 02138, USA \\ ${ }^{2}$ NASA Goddard Space Flight Center, Greenbelt, MD 20771, USA \\ ${ }^{3}$ Villanova University, Villanova, PA 19085 USA
}

\begin{abstract}
A wideband profiled corrugated feedhorn was developed for multichroic applications. This feedhorn features a return loss of better than $-25 \mathrm{~dB}$ and cross polarization peaks below $-30 \mathrm{~dB}$, over a fractional bandwidth of $>50 \%$. Its performance is close to that of the ring-loaded corrugated feedhorn; however, the design presented is much easier to fabricate at millimeter wavelengths.
\end{abstract}

\section{INTRODUCTION}

$\mathrm{F}$ eedhorns are widely used in the millimeter and $\mathrm{THz}$ frequency bands for radiation coupling. In multichroic applications, the feedhorn is required to have low return loss over a wide frequency range and its beam patterns should be symmetrical in the $\mathrm{E}$ - and the $\mathrm{H}$ - planes, while presenting very low sidelobe levels. A classical design used to achieve these design goals is the ring-loaded corrugated feedhorn [1]. This type of feedhorn has been made to provide highly symmetrical beam pattern with good return loss for a bandwidth larger than a full waveguide bandwidth. However, the ring structures in these feedhorn are extremely difficulty to fabricate, especially for high frequency operation.

A profiled corrugated feedhorn design is an alternative to the ring-loaded feedhorn design. Figure 1 shows the profile of our profiled corrugated feedhorn designed to cover two separate bands centered on $150 \mathrm{GHz}$ and $220 \mathrm{GHz}$. The design process employed a mode-matching method [2] for the beam pattern calculation. The angular responses in Figure 2 were calculated from the modal content at the feedhorn aperture by matching the boundary conditions and cascading the response for each groove section in the feed structure. We used a straight flare corrugated horn with a TBS opening angle and a variable pitch in mode launching section as the initial input profile [3]. The input waveguide diameter of the initial horn was $0.804 \mathrm{~mm}$, corresponding to a cut off frequency of 109.24 $\mathrm{GHz}$ for the TE11 mode. We used the Powell method [4] as the optimization algorithm to generate the desired profile. During optimization, we kept the input waveguide, groove dimensions and the total length of the initial corrugated horn fixed and constructed new profiles using the natural profile method [5]. The slot width was held constant during optimization. An insightful discussion of the influence of the slot geometry on the cross polarization performance of a corrugated feedhorn can be found in [6]. For the final design, slot width and period are TBS in units of the feed structure's $\mathrm{HE}_{11}$ hybrid wavelength $\lambda_{x}[6]$. The slot depths range from $\sim 0.5 \lambda_{x}$ to $0.25 \lambda_{x}$ in the throat to flare section respectively. The profile shape, pitch locations in the throat region and the aperture size of the horn were free parameters to be optimized. We used a similar penalty function as that described in [5]:

$$
\chi^{2}=\sum_{i=1}^{N} \sum_{j=1}^{3}\left\{\alpha_{j}\left[\Delta_{j}\left(f_{i}\right)\right]^{2}\right\}
$$

where $i$ is the index for the sum over a discrete set of frequencies, $j$ is the index for the sum over penalty function parameters and $\alpha_{j}$ is the $j$ th parameter weight. In our optimization, $N=101$ (from $140 \mathrm{GHz}$ to $240 \mathrm{GHz}$, $\Delta f=1.0 \mathrm{GHz}$ ) and penalty function parameters are cross polarization, return loss and the FWHM of the beam. The explicit forms for $\Delta_{j}$ are:

$$
\begin{aligned}
& \Delta_{1}(f)= \begin{cases}X P(f)-X P_{0} & \text { if } X P(f)>X P_{0} \\
0 & \text { if } X P(f)<X P_{0},\end{cases} \\
& \Delta_{2}(f)= \begin{cases}R L(f)-R L_{0} & \text { if } R L(f)>R L_{0} \\
0 & \text { if } R L(f)<R L_{0},\end{cases} \\
& \Delta_{3}(f)=F W H M(f)-F W H M_{0},
\end{aligned}
$$

where $X P(f), R L(f)$ and $F W H M(f)$ are the peaks of cross polarization, return loss and FWHM of the beam pattern at frequency $f$, respectively. $X P_{0}=-35 d B, R L_{0}=-30 d B$ and $F W H M_{0}=9.0 \mathrm{deg}$ are the thresholds of the optimization. If the cross polarization and return loss are lower than the optimization thresholds, they will be omitted from the penalty function.

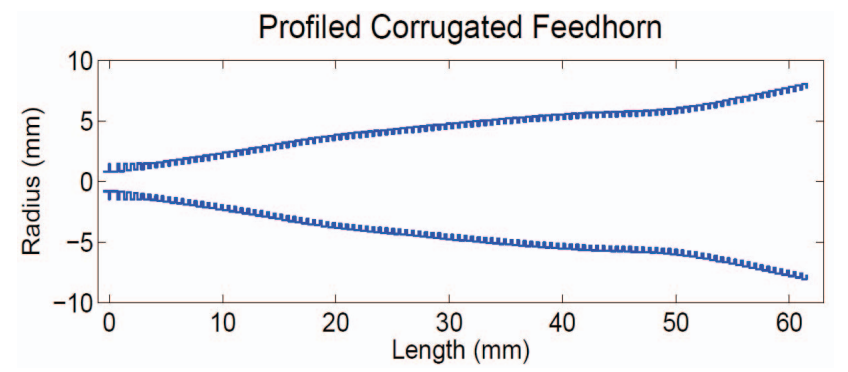

Figure 1. The profile of our profiled corrugated feedhorn. This feedhorn is optimized for operation in a $150 \mathrm{GHz}$ and $220 \mathrm{GHz}$ multichroic focal plane.

\section{RESULTS}

Figure 2 shows beam patterns from $150 \mathrm{GHz}$ to $220 \mathrm{GHz}$. This feedhorn is designed to have a FWHM angle of about 9 degrees at center frequency. The sidelobe level is below -30 $\mathrm{dB}$ and the peaks of cross polarization are below -45 . The feedhorn beam properties are listed in Table 1. This feedhorn has an average antenna gain of about $26 \mathrm{~dB}$ and about $45 \%$ aperture efficiency. Figure 3 shows the peak cross polarization level and return loss of the horn between $140 \mathrm{GHz}$ and 240 GHz. We use Ludwig's third definition [7] for cross polarization and the cross polarization result represents the peak values along the azimuthal angle at each frequency. The 
plot shows that the cross polarization is below $-30 \mathrm{~dB}$ over a $60 \%$ fractional bandwidth, while the return loss, which is limited by the input waveguide is below $-25 \mathrm{~dB}$ over a $50 \%$ bandwidth. This profiled corrugated horn has much better bandwidth performance compared to the conventional corrugated feedhorn and is a strong candidate form wide band multichroic applications.
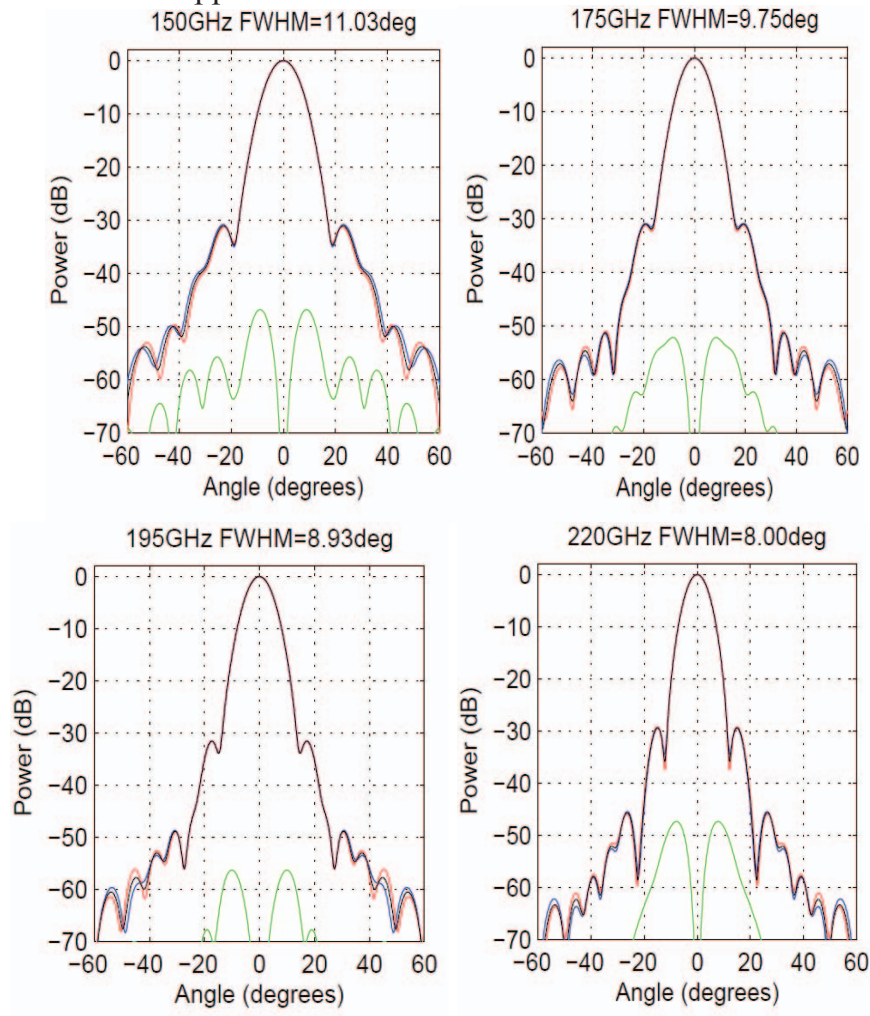

Figure 2. The beam patterns of profiled corrugated feedhorn from $150 \mathrm{GHz}$ to $220 \mathrm{GHz}$. E plane is in blue, $\mathrm{H}$ plane is in red, diagonal (co-polarization) plane is in black and cross polarization plane is in green. The sidelobe levels are below $-30 \mathrm{~dB}$ and peaks of the cross polarization are below $-45 \mathrm{~dB}$.

Table 1. Feedhorn Beam Property Summary.

\begin{tabular}{c|ccc}
\hline $\begin{array}{c}\text { Frequency } \\
{[\mathrm{GHz}]}\end{array}$ & $\begin{array}{c}\text { Beam Solid } \\
\text { Angle } \\
{[\mathrm{Sr}]}\end{array}$ & $\begin{array}{c}\text { Antenna } \\
\text { Gain } \\
{[\mathrm{dB}]}\end{array}$ & $\begin{array}{c}\text { Aperture } \\
\text { Efficiency } \\
{[\%]}\end{array}$ \\
\hline 140 & 0.0490 & 24.09 & 49.93 \\
150 & 0.0435 & 24.60 & 48.99 \\
160 & 0.0396 & 25.02 & 47.39 \\
170 & 0.0361 & 25.42 & 45.98 \\
180 & 0.0331 & 25.80 & 44.78 \\
190 & 0.0302 & 26.19 & 43.94 \\
200 & 0.0278 & 26.55 & 43.12 \\
210 & 0.0256 & 26.91 & 42.47 \\
220 & 0.0230 & 27.37 & 43.08 \\
230 & 0.0209 & 27.78 & 43.32 \\
\hline
\end{tabular}

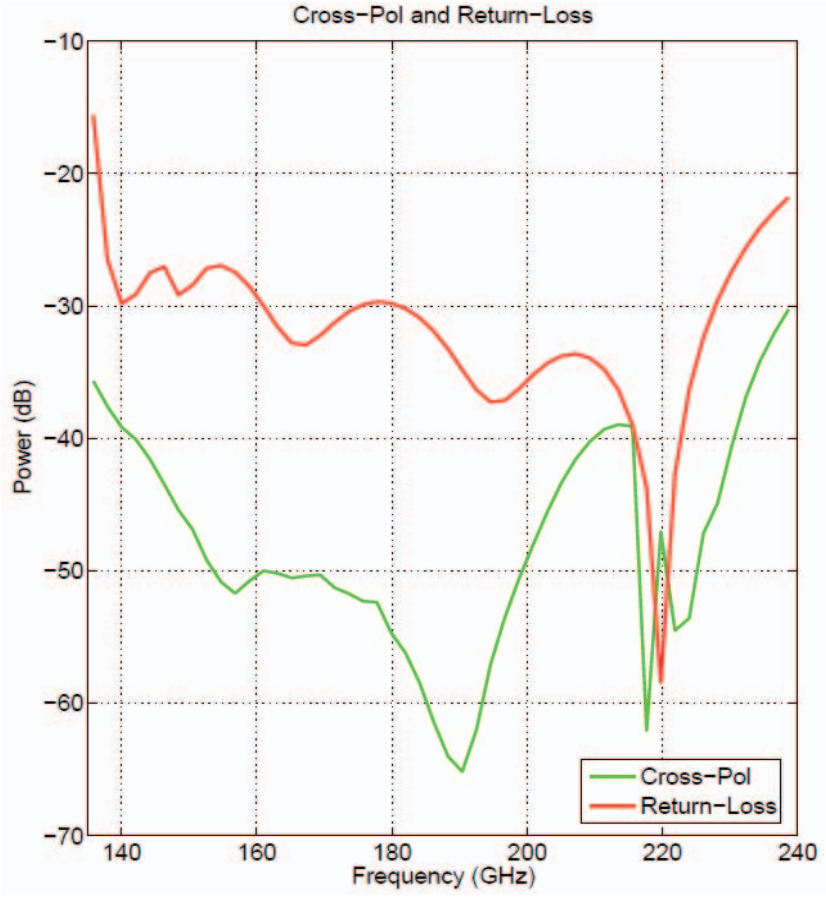

Figure 3. The peak cross polarization level and return loss of the designed feedhorn. Cross polarization is below $-35 \mathrm{~dB}$ and the return loss is below -25 $\mathrm{dB}$ between 140 and $230 \mathrm{GHz}$ (50\% fractional bandwidth).

\section{DISCUSSIONS}

Compared to the ring loaded corrugated horn, this profiled corrugated feedhorn can be fabricated more easily using the platelet technique [8]. A large number of feedhorns can be made in parallel by milling platelets and stacking them in layers. The relative ease of fabrication and its wide bandwidth makes it a good candidate for large format multichroic focal plane applications at millimeter wave frequencies.

\section{REFERENCES}

[1] Yoshiro, T., Tsutomv, M. and Fumio, T., 'The ring-loaded corrugated waveguide', Trans. IEEE, 1971, MTT-19, pp.947-950

[2] G. L. James. 'Analysis and design of TE11-to-HE11 corrugated cylindrical waveguide mode converters'. IEEE Transactions on Microwave Theory Techniques, 29:1059-1066, October 1981.

[3] X Zhang, "Design of conical corrugated feed horns for wide-band highfrequency applications," Microwave Theory and Techniques, IEEE Transactions on, vol. 41, no. 8, pp.1263,1274, Aug 1993

[4] W. H. Press, S. A. Teukolsky, W. T. Vetterling, and B. P. Flannery. 'Numerical recipes in C'. The art of scientific computing. Cambridge: University Press, c1992, 2nd ed., 1992.

[5] L. Zeng, C. L. Bennett, D. T. Chuss, and E. J. Wollack. 'A Low CrossPolarization Smooth-Walled Horn With Improved Bandwidth'. IEEE Transactions on Antennas and Propagation, 58:1383-1387, April 2010.

[6] P.J.B. Clarricoats and A.D. Olver, "Corrugated Horns for Microwave Antennas", 1984, Peter Pergrinus, New York.

[7] A. C. Ludwig. 'The definition of cross polarization'. IEEE Transactions on Antennas and Propagation, 21:116-119, 1973.

[8] R.W. Haas, et al., "Fabrication and Performance of MMW and SMMW Platelet Horn Arrays," 1993, International Journal of Infrared and Millimeter Waves, Vol. 14, pp. 2289-2293. 